\title{
Branched-chain and aromatic amino acids in relation to behavioral problems among young Inuit from Nunavik, Canada: a cohort study
}

\author{
Audray St-Jean ${ }^{1}$, Salma Meziou ${ }^{1}$, Cynthia Roy ${ }^{2}$, Pierre Ayotte ${ }^{2}$, Gina Muckle ${ }^{3}$ and Michel Lucas ${ }^{4}$
}

BACKGROUND: Obesity and insulin resistance are linked with mood disorders, and elevated concentrations of branchedchain (BCAAs) and aromatic amino acids (AAAs). Our study aimed to prospectively assess the relationship between childhood plasma BCAAs and AAAs, and behavioral problems in young Inuit from Nunavik.

METHODS: We analyzed data on 181 children (with a mean age of 11.4 years at baseline) involved in the Nunavik Child Development Study. Plasma BCAA and AAA concentrations were measured in childhood (2005-2010). BCAA/AAA tertiles - the ratio of total BCAAs to AAAs - were considered as surrogate categorical independent variables. Behavioral problems were assessed with the Youth Self-Report (YSR) from the Child Behavior Checklist about 7 years later during adolescence (2013-2016). ANOVA ascertained relationships between BCAA/AAA tertiles and YSR outcomes.

RESULTS: Ascending BCAA/AAA tertiles were positively associated $\left(P_{\text {trend }}<0.05\right)$ with somatic complaint scores. Scores of somatic complaints syndrome were significantly higher ( $\left.P_{\text {trend }}<0.05\right)$ with increasing BCAA/AAA tertiles among both normal and overweight/obese participants.

CONCLUSION: Our results suggest that higher BCAA/AAA ratios in childhood are significantly associated with somatic complaints in adolescence.

O besity among children and adolescents is a major public health issue with an increased risk of metabolic and cardiovascular complications, as well as premature mortality, in adulthood (1). Overweight and obesity are associated with significant mood disorders throughout life span (2-6). Obesity is also linked with internalizing problems in youth (7-9), but data supporting this relation with externalizing problems are limited $(10,11)$.

Several studies have reported that obesity and insulin resistance among adults are characterized by a prominent metabolomic signature, especially elevation of amino acid (AA) blood levels, such as branched-chain amino acids (BCAAs: isoleucine, leucine, and valine) and aromatic amino acids (AAAs: phenylalanine and tyrosine) (12-15). Augmented concentrations of these metabolomic biomarkers predicted the risk of future diabetes in longitudinal studies of adults (16-18). BCAAs were also noted in obese children $(19,20)$ and prospectively associated with insulin resistance in youth (21). The data on obese adolescents remain inconsistent $(22,23)$.

BCAA uptake into the central nervous system is competitive with respect to AAAs. BCAAs are transported through the blood-brain barrier by L-type or large amino acid transporter 1 (LAT1) (24). LAT1 is shared by several large neutral amino acids, including both BCAAs and AAAs, which compete for access. Thus, elevation of circulating BCAAs could decrease AAA uptake into the central nervous system (25). Both tyrosine and phenylalanine are substrates for catecholamine synthesis (dopamine, norepinephrine, and epinephrine), whereas tryptophan is a serotonin precursor. These neurotransmitters are known for their roles in behavioral functions. A variation in AAA concentrations could affect their brain levels and subsequently neurotransmitter synthesis (26). To our knowledge, no previous study has investigated the potential impact of AAAs on behavior in humans. A study in rats showed increased stress/anxiety in animals fed high-energy diets supplemented with BCAAs compared with controls (27). They also noted decreased tyrosine and tryptophan concentrations in brain tissues.

In Canada, the prevalence of obesity and diabetes is higher among the Indigenous population compared with that in the non-Indigenous population (28). Given the emerging association between BCAAs and AAAs with obesity and insulin resistance, as well as the potential impact of AAAs on behavior, the hypothesis that these metabolomic biomarkers could be implied with behavioral modifications deserves consideration. This would allow extending the actual knowledge of the metabolism related to obesity and insulin resistance on behavior. Using data on a longitudinal cohort of young Inuit from Nunavik (Canada), we explored the relationship between metabolomic biomarkers during childhood and self-reported behavioral problems at adolescence.

\footnotetext{
'Population Health and Optimal Health Practices Research Unit, CHU de Québec_-Université Laval, Québec, Canada; ${ }^{2}$ Institut National de Santé Publique du Québec (INSPQ), Québec, Canada; ${ }^{3}$ School of Psychology, Université Laval, Québec, Canada; ${ }^{4}$ Department of Social and Preventive Medicine, Université Laval, Québec, Canada. Correspondence: Michel Lucas, (michel.lucas@crchuq.ulaval.ca) 
We hypothesized that elevated BCAA concentrations-a surrogate of initial obesity and/or insulin resistance statescould lead to a decrease in AAA uptake into the central nervous system resulting in behavioral modifications. Because obesity might be involved in the causal chain between BCAAs and behavioral problems, we assessed the potential effect modification of baseline BMI status in this relation.

\section{METHODS}

\section{Study Design and Population}

Data were sourced from the Nunavik Child Development Study (NCDS). Participants were young Inuit from Nunavik, a region located north of the 55th parallel in northern Québec, Canada. The study design has been described previously (29). Briefly, the NCDS is a follow-up of youth recruited before birth when their mothers participated in the Cord Blood Monitoring Program (1993-1998) (30) or the Environmental Contaminants and Child Development Study (1996-2000) (31). Individuals who participated in the 20052010 school-age (baseline for the current study) and 2013-2016 adolescent (follow-up) surveys were eligible for inclusion in the present investigation. Of the 289 participants eligible for follow-up, 212 were recruited (participation rate $=73.4 \%$ ). Reasons for not participating in the follow-up were formal refusal, moving out of Nunavik region, and loss to follow-up or death. Participants with missing data on AA quantification $(n=28)$, behavioral assessment $(n=2)$, or both $(n=1)$ were excluded, which left 181 participants for the current analysis. Participants came from 14 villages but assessments took place in the 3 largest Nunavik villages: Inukjuak, Kuujjuaq, and Puvirnituq. Participants who resided in other communities were transported by plane for testing. Anthropometric data were recorded by research nurses using standard measurement procedures. A maternal interview was conducted at baseline to gather information on sociodemographic background. Each child provided a venous blood sample $(20 \mathrm{ml})$, which was kept frozen at $-80^{\circ} \mathrm{C}$ in Nunavik and transported by plane to Québec City (Canada) for biological analysis. During adolescent follow-up assessment, behavioral questionnaires were administered by a research professional accompanied by an interpreter, when needed.

Participation was voluntary and subject to informed consent. At baseline, each child's primary caregiver provided written consent, with oral assent provided by each child; written consent was obtained at adolescence. Consent and assent forms were approved by the Nunavik Nutrition and Health Committee, and the research ethics review board of CHU de Québec Research Center and Wayne State University.

\section{Measurements}

Behavioral assessment. The Youth Self-Report (YSR) from the Child Behaviour Checklist Tests battery (32) was administered during the adolescent follow-up period. The YSR contains 112 items scored as follows: $0=$ not true, $1=$ somewhat or sometimes true, or $2=$ very true or often true, applicable to the participant within the past 6 months. Eight syndrome subscales are computed by summing the scores on the following specific items: anxious/depressed, withdrawn/depressed, somatic complaints, rule-breaking behavior, aggressive behavior, attention problems, social problems, and thought problems. Total problems score is based on the sum of eight syndromes and other problems. Internalizing problems score is the sum of anxious/depressed, withdrawn/depressed, and somatic complaints, whereas rule-breaking and aggressive behaviors are summed to compute the externalizing problems score. We restricted our analyses to internalizing, externalizing, and attention problems to limit the number of statistical analyses. Raw scores were considered for each YSR scale because no normative data were available for the Inuit youth.
$\boldsymbol{A} \boldsymbol{A}$ quantification. $\mathrm{BCAA}$ and $\mathrm{AAA}$ concentrations (mg/l) were measured at baseline. Nonfasting plasma concentrations of AAs were quantified at the Centre de Toxicologie du Québec, Institut National de Santé Publique, Québec City (Canada), by isotope-dilution liquid chromatography/hybrid quadrupole-time-of-flight-mass spectrometry. The complete assay protocol is described elsewhere (33). Inter-day precision (coefficient of variation) of the method was $<15 \%$, and trueness ranged from 0.5 to $5.5 \%$.

Weight status. Weight was measured without shoes on a digital balance, and height was recorded without shoes with a stadiometer. Two measurements were taken for each parameter, and a third was obtained if discrepancy occurred between them (weight $>500 \mathrm{~g}$ and height $>0.5 \mathrm{~cm}$ ). Final values were based on the average of the two closest measurements. BMI was calculated by dividing weight $(\mathrm{kg})$ by height squared $\left(\mathrm{m}^{2}\right)$. Weight status was defined at baseline according to the International Obesity Task Force (IOTF) system. In a previous study (34), we noted that IOTF was more specific in assessing obesity in our population. The IOTF classification is based on extrapolation of adult BMI cutoff points for overweight $\left(25 \mathrm{~kg} / \mathrm{m}^{2}\right)$ and obesity $\left(30 \mathrm{~kg} / \mathrm{m}^{2}\right)$ at the age of 18 years (35). Normal weight described participants who were neither overweight nor obese. BMI $z$-scores were calculated using the Centers for Disease Control and Prevention SAS program (36).

Family environment at baseline. Socioeconomic status score was assessed with the Hollingshead index, computed from predefined scores given for parental occupational status and education (37). Residential crowding was defined as the number of persons living in the house per room. Food insecurity was defined as the mother reporting at least 1 day without sufficient food or funds to purchase food in the month preceding the interview (yes/no).

\section{Data Analyses}

AA concentrations $(\mathrm{mg} / \mathrm{l})$ were converted into micromolar concentrations $(\mu \mathrm{mol} / \mathrm{l})$. Because BCAA uptake into the central nervous system is competitive with respect to AAAs, we created BCAA/AAA ratios by dividing total $\mathrm{BCAA}$ concentrations by AAAs. Baseline descriptive characteristics of the study participants are presented according to BCAA/AAA tertile categories. Data are reported as arithmetic means \pm SD for continuous variables, and proportions $(n, \%)$ for categorical variables. Descriptive statistics for YSR syndrome scores at adolescent follow-up are given as means $\pm S D$ and medians (minimum-maximum). Multivariate-adjusted means \pm SEM of YSR scores by BCAA/AAA tertiles were obtained with general linear models. Means across tertiles were compared by ANCOVA. The Bonferroni correction was used for multiple comparisons tests-i.e., comparisons of syndrome scores between tertiles. Considering the number of comparisons for the multiple syndrome subscales (eight models), we further evaluated statistical significance using the conservative Bonferroni $P$ value $(0.05-$ $/ 8=0.00625$ ) to control family-wise error rate. Tests for linear trends across tertiles were assessed with the SAS software PROC GLM CONTRAST by assigning the median value of each tertile for continuous variables and with the Cochran-Armitage test for trend for categorical variables. Potential confounders, identified a priori and included in adjusted models, were baseline age (continuous), sex, socioeconomic status (continuous), and food insecurity (dichotomous). Single imputation completed missing data on food insecurity $(n=1)$. We also assessed potential effect modification of baseline BMI status (normal weight or overweight/obese) in the association between BCAA/AAA tertiles and mean YSR scores. Interaction terms between the BCAA/AAA ratio and the weight group were added into linear models to test whether interaction was significant. Statistical analyses were conducted with SAS software (version 9.4, SAS Institute, Cary, NC). Two-sided $P<0.05$ values were considered to be statistically significant. 
Articles | St-Jean et al.

Table 1. Descriptive characteristics and statistics ${ }^{\mathrm{a}}: \mathrm{YSR}^{\mathrm{b}}$ syndrome scores of the study sample according to BCAA/AAA tertiles, Nunavik, Canada $(n=181)$

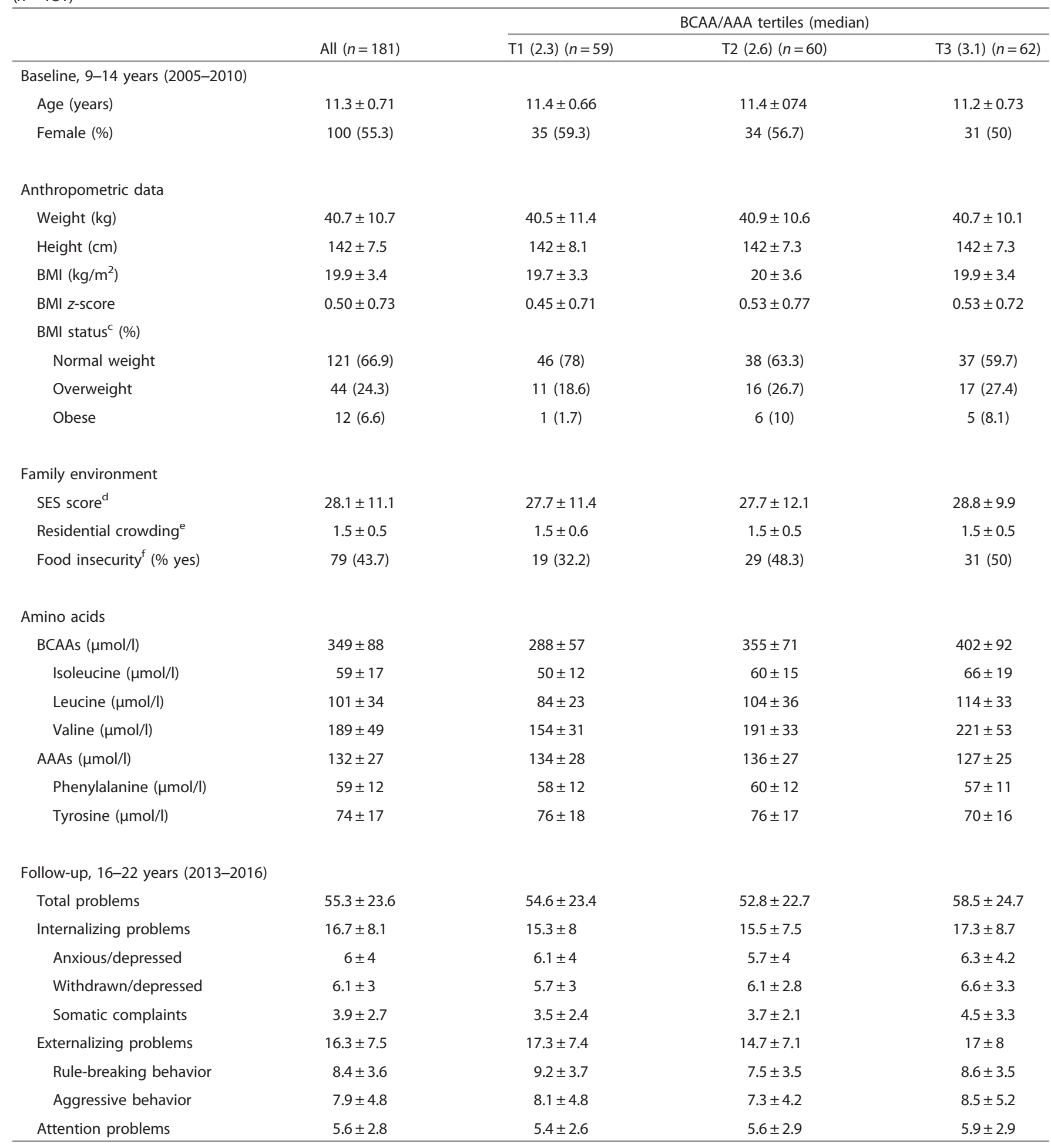

AAAs, aromatic amino acids; BCAAs, branched-chain amino acids; IOTF, International Obesity Task Force; SES score, socioeconomic status score; YSR, Youth Self-Report.

aValues are presented as means \pm SD or $n(\%)$. Information was missing for four participants on weight, height, BMI, BMI $z$-score, and BMI status, and for one participant on residential crowding and food insecurity.

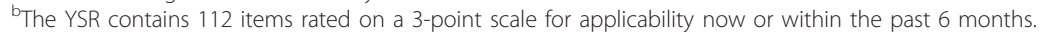

${ }^{\mathrm{c}}$ According to the IOTF classification.

${ }^{\mathrm{d}}$ Assessed with the Hollingshead index.

${ }^{\mathrm{e}}$ Number of persons/room.

fDefined as the mother reporting not having enough food for her family at least 1 day in the preceding month. 


\section{RESULTS}

Table 1 summarizes the baseline descriptive characteristics of study participants according to BCAA/AAA tertiles. The medians of the tertiles ( $\min$., max.) were, respectively, 2.3 (1.7, 2.4), $2.6(2.4,2.7)$, and $3.1(2.8,4.8)$. At baseline, participants were aged between 9 and 14 years $($ mean $=11.3, \mathrm{SD}=0.71$ ), and $55.3 \%$ were girls. Mean concentrations of BCAAs and AAAs were, respectively, $349(\mathrm{SD}=88)$ and $132(\mathrm{SD}=27)$ $\mu \mathrm{mol} / \mathrm{l}$. Descriptive statistics on YSR syndrome scores assessed during adolescence are also presented. At followup, participants were between the ages of 16 and 22 years (mean $=18.4, \mathrm{SD}=1.1$ ). Average follow-up time was 7.1 years $(\mathrm{SD}=1.2)$. Total problems mean score was $55.3(\mathrm{SD}=23.6)$. Mean score was $16.7(\mathrm{SD}=8.1)$ for internalizing, 16.3 $(\mathrm{SD}=7.5)$ for externalizing, and $5.6(\mathrm{SD}=2.8)$ for attention problems.

Table 2 describes the adjusted mean scores of YSR syndromes at adolescent follow-up according to BCAA/ AAA tertiles. We observed that somatic complaints scores were significantly higher $\left(P_{\text {trend }}<0.05\right)$ with rising BCAA/ AAA tertiles. Internalizing problems $\left(P_{\text {trend }}=0.05\right)$ and withdrawn/depressed $\left(P_{\text {trend }}=0.06\right)$ scores showed a similar pattern, but were not statistically significant. No significant differences in syndrome scores across tertiles were found. Table 3 depicts adjusted mean scores of YSR syndrome scores by BCAA/AAA tertiles stratified for baseline BMI status. Interaction terms between metabolomic biomarker concentrations (BCAA/AAA ratio) and weight status (normal weight or overweight/obese) were not significant (all $P>0.14$ ). Among overweight or obese participants, but not among normal-weight participants, ascending BCAA/ AAA tertiles were marginally related to withdrawn/depressed scores $\left(P_{\text {trend }}=0.08\right)$. Internalizing problems showed a similar pattern among both normal-weight $\left(P_{\text {trend }}=0.12\right)$ and overweight/obese $\left(P_{\text {trend }}=0.06\right)$ participants. Somatic complaints scores were significantly higher $\left(P_{\text {trend }}<0.05\right)$ with rising
BCAA/AAA tertiles among both normal-weight and overweight/obese participants.

Additional analyses using other possible types of indicators were also conducted (data not shown). Results using BCAAs and AAAs independently in a same model were not significant, but it does not take into account the competitive mechanism through which these metabolites are transported from the blood to the brain. The use of a proportion of BCAAs over the sum of BCAAs plus AAAs led to similar results as the $\mathrm{BCAA} / \mathrm{AAA}$ ratio.

\section{DISCUSSION}

Our study is the first to explore the relationship between plasma BCAAs and AAAs and behavioral problems. Our data, on this cohort of young Inuit from Nunavik, are somehow consistent with our hypothesis that elevated plasma concentrations of metabolomic biomarkers-a surrogate of initial obesity and/or insulin resistance states - could be associated with behavioral problems. Higher mean scores of somatic complaints were observed with ascending BCAA/AAA tertiles. We found no significant linear trends for externalizing and attention problems. Stratification for BMI status did not reveal significant relations among overweight/obese participants only for somatic complaints scores $\left(P_{\text {trend }}=0.03\right)$. Associations with internalizing problems $\left(P_{\text {trend }}=0.06\right)$ and withdrawn/depressed $\left(P_{\text {trend }}=0.08\right)$ scores were close to significance. Among normal-weight participants, scores of somatic complaints syndrome were significantly higher $\left(P_{\text {trend }}\right.$ $<0.05)$ with increasing BCAA/AAA tertiles.

To date, a single animal study (27) assessed the relationship between metabolomic biomarkers and behavioral problems. The results of Coppola et al. (27) are consistent with our observations. A decrease in the plasma ratio of AAAs on the sum of all BCAAs and AAAs was observed in rats fed highenergy diets supplemented with BCAAs compared with controls. Increased stress/anxiety was also seen. In these experiments, plasma AAAs correlated with brain tissue levels,

Table 2. Mean YSR syndrome scores ${ }^{\mathrm{a}}$ at adolescence according to baseline BCAA/AAA tertiles during childhood

\begin{tabular}{|c|c|c|c|c|}
\hline & \multicolumn{4}{|c|}{ BCAA/AAA tertiles } \\
\hline & $\mathrm{T} 1(2.3)(n=59)$ & $\mathrm{T} 2(2.6)(n=60)$ & T3 (3.1) $(n=62)$ & $P_{\text {trend }}$ \\
\hline Internalizing problems & $15.1 \pm 1$ & $15.3 \pm 1$ & $17.7 \pm 1$ & 0.05 \\
\hline Withdrawn/depressed & $5.7 \pm 0.4$ & $6 \pm 0.4$ & $6.7 \pm 0.4$ & 0.06 \\
\hline Somatic complaints & $3.5 \pm 0.3$ & $3.6 \pm 0.3$ & $4.6 \pm 0.3$ & 0.02 \\
\hline Rule-breaking behavior & $9.2 \pm 0.5$ & $7.4 \pm 0.5$ & $8.6 \pm 0.5$ & 0.55 \\
\hline Aggressive behavior & $8 \pm 0.6$ & $7.3 \pm 0.6$ & $8.5 \pm 0.6$ & 0.50 \\
\hline Attentions problems & $5.4 \pm 0.4$ & $5.6 \pm 0.4$ & $5.9 \pm 0.4$ & 0.29 \\
\hline
\end{tabular}




\section{Articles | St-jean et al.}

Table 3. Mean scores of YSR syndrome scores ${ }^{a}$ at adolescence according to baseline BCAA/AAA tertiles and BMI status ${ }^{b}$ during childhood

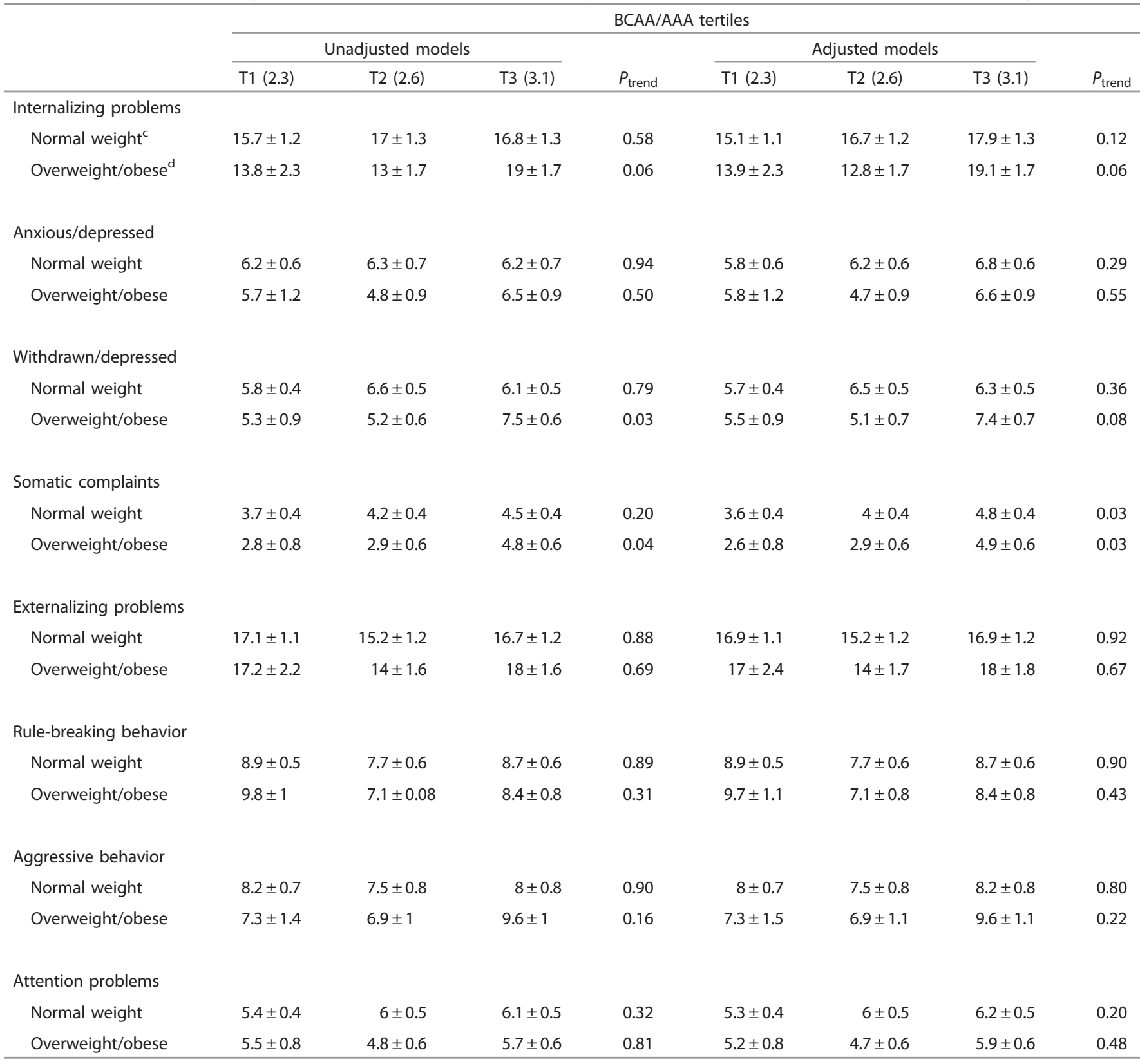

AAAs, aromatic amino acids; BCAAs, branched-chain amino acids; IOTF, International Obesity Task Force; YSR, Youth Self-Report.

a Values are presented as adjusted means \pm SEM. Unadjusted models include the YSR syndrome scores and the BCAA/AAA tertiles variables, whereas adjusted models include also baseline age, sex, socioeconomic status, and food insecurity.

bBMI status at baseline according to IOTF classification, missing for four participants.

${ }^{c}$ Normal-weight $\mathrm{T} 1 n=46, \mathrm{~T} 2 n=38, \mathrm{~T} 3 n=37$.

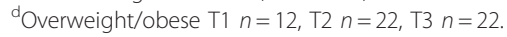

and reduced tyrosine and tryptophan values were recorded. The authors also discerned significantly diminished serotonin concentrations in the frontal cortex of BCAA-fed rats, but not norepinephrine and dopamine. This study provides a possible biological explanation of our results-i.e., the competitive mechanism of BCAAs on AAA uptake into the central nervous system. BCAAs and AAAs both share the same carrier and compete for access, so that elevation of circulating BCAAs is predicted to lower AAA uptake. Therefore, we could speculate that increased plasma BCAA/AAA ratios would be expected to decrease AAA uptake through the blood-brain barrier. This might explain why higher YSR scores were observed in the highest BCAA/AAA tertile. Phenylketonuria is a pathological example of this effect, and is characterized by cognitive dysfunction. When the disease is left untreated, higher phenylalanine concentrations influence the transport of other large neutral amino acids at the bloodbrain barrier. Phenylalanine entry into the brain is increased, 
whereas other AAs are restricted (38). Decreased AAA concentrations could affect serotonin and catecholamine neurotransmitter synthesis, which have been implicated in the pathogenesis of depression $(39,40)$.

Obesity is known to be associated with mood disorders $(2,3)$ and elevated metabolomic biomarker concentrations $(12,13,19-21)$, which suggest that obesity could be involved in the causal chain between BCAAs and YSR syndrome scores. However, we found only one significant association between metabolomic biomarkers and behavioral problems among participants categorized as overweight or obese. YSR mean scores tend to be higher with increasing BCAA/AAA tertiles for scores of internalizing problems $\left(P_{\text {trend }}=0.06\right)$, withdrawn/depressed $\left(P_{\text {trend }}=0.08\right)$, and somatic complaints $\left(P_{\text {trend }}=0.03\right)$. A significant trend was only observed for somatic complaints scores across both normal weight and overweight/obese strata. These results among overweight/ obese strata are consistent with our hypothesis that obesity might be involved in the causal chain between BCAAs and behavioral problems. Previous studies have noted an association between obesity and internalizing problems in youth (7-9), but data on relation with externalizing problems are limited $(10,11)$. An insufficient number of individuals classified as overweight or obese could be an explanation to why we found only one significant result among overweight/ obese strata. We hypothesize that our significant results observed among normal-weight participants could be explained by the biological changes occurring before weight gain. In addition, even if elevated BCAA and AAA concentrations are strongly associated with obesity, other factors may be involved in obesity, which itself could lead to internalizing symptoms through various pathways, implying low selfesteem, experiences of shame, body dissatisfaction, and/or poor body weight perception.

This study must be interpreted in light of its limitations because of its exploratory nature and statistical power. First, our sample size was relatively small. Statistical power was perhaps insufficient for stratified analysis by BMI status, especially in overweight/obese strata. The use of a more conservative $P$ value would have been preferable, but because of our small sample size $P<0.05$ was used. Hence, the results should be interpreted with caution. Second, our venous blood samples were not collected under fasting conditions, which affect the accuracy of our exposition measurement and could lead to nondifferential misclassification, resulting in bias toward null hypothesis. Third, our unique racial and cultural Inuit population may limit the generalization of findings to other populations. Other studies looked at BCAAs and AAAs in relation to obesity and/or insulin resistance in youth (19-21). However, concentration values are not presented, which does not permit comparisons with our results. In addition, the fact that our blood samples were not collected under fasting conditions is a limitation. Despite these limitations, the study has several strengths, including longitudinal analysis, which reduced the risk of reverse causation compared with the cross-sectional investigation, and assessment of behavioral problems through a state-of-the-art self-reported questionnaire rather than by parent proxy. Weight and height were measured directly, decreasing the underestimation of overweight and obesity prevalence.

In summary, our study is the first to explore the association between metabolomic biomarkers and behavioral problems among humans. The results suggest that higher BCAA/AAA ratios in childhood are prospectively linked with self-reported behavioral problems in adolescence, especially somatic complaints.

\section{ACKNOWLEDGMENTS}

We are grateful to the Nunavik population, particularly the parents and youth, who participated in this study.

\section{AUTHOR CONTRIBUTIONS}

G.M. was principal investigator of the Nunavik Child Development Study. A.S.J., S.M., C.R., P.A., G.M., and M.L. conceptualized the current analysis. A.S. J. analyzed the data and wrote the first manuscript draft. All authors contributed to results interpretation and critical manuscript revision for intellectual content and approved the final text version.

\section{STATEMENT OF FINANCIAL SUPPORT}

This research was supported by grants from the Canadian Institutes of Health Research, the National Institutes of Health/National Institute of Environmental Health Sciences; Northern Contaminants Program, Indian and Northern Affairs Canada; Health Canada; Hydro-Québec (Environmental Child Initiative); and the Joseph Young, Sr., Fund, State of Michigan. The funding sources were not involved in data analysis, manuscript writing, and publication.

Disclosure: The authors declare no conflict of interest.

\section{REFERENCES}

1. Reilly JJ, Kelly J. Long-term impact of overweight and obesity in childhood and adolescence on morbidity and premature mortality in adulthood: systematic review. Int J Obes 2011;35:891-8.

2. de Wit L, Luppino F, van Straten A, Penninx B, Zitman F, Cuijpers P. Depression and obesity: a meta-analysis of community-based studies. Psychiatry Res 2010;178:230-5.

3. Gariepy G, Nitka D, Schmitz N. The association between obesity and anxiety disorders in the population: a systematic review and metaanalysis. Int J Obes 2010;34:407-19.

4. Luppino FS, de Wit LM, Bouvy PF, et al. Overweight, obesity, and depression: a systematic review and meta-analysis of longitudinal studies. Arch Gen Psychiatry 2010;67:220-9.

5. Pan A, Sun Q, Czernichow S, et al. Bidirectional association between depression and obesity in middle-aged and older women. Int J Obes 2012;36:595-602.

6. Sanderson K, Patton GC, McKercher C, Dwyer T, Venn AJ. Overweight and obesity in childhood and risk of mental disorder: a 20-year cohort study. Aust N Z J Psychiatry 2011;45:384-92.

7. Bradley RH, Houts R, Nader PR, O'Brien M, Belsky J, Crosnoe R. The relationship between body mass index and behavior in children. J Pediatr 2008;153:629-34.

8. Goldfield GS, Moore C, Henderson K, Buchholz A, Obeid N, Flament MF. Body dissatisfaction, dietary restraint, depression, and weight status in adolescents. J Sch Health 2010;80:186-92.

9. ter Bogt TF, van Dorsselaer SA, Monshouwer K, Verdurmen JE, Engels RC, Vollebergh WA. Body mass index and body weight perception as risk factors for internalizing and externalizing problem behavior among adolescents. J Adolesc Health 2006;39:27-34. 


\section{Articles | St-jean et al.}

10. Mustillo S, Worthman C, Erkanli A, Keeler G, Angold A, Costello EJ. Obesity and psychiatric disorder: developmental trajectories. Pediatrics 2003;111:851-9.

11. Pervanidou P, Bastaki D, Chouliaras G, Papanikolaou K, Kanaka-Gantenbein C, Chrousos G. Internalizing and externalizing problems in obese children and adolescents: associations with daily salivary cortisol concentrations. Hormones 2015;14:623-31.

12. Huffman KM, Shah SH, Stevens RD, et al. Relationships between circulating metabolic intermediates and insulin action in overweight to obese, inactive men and women. Diabetes Care 2009;32:1678-83.

13. Newgard CB, An J, Bain JR, et al. A branched-chain amino acid-related metabolic signature that differentiates obese and lean humans and contributes to insulin resistance. Cell Metab 2009;9:311-26.

14. Tai ES, Tan ML, Stevens RD, et al. Insulin resistance is associated with a metabolic profile of altered protein metabolism in Chinese and AsianIndian men. Diabetologia 2010;53:757-67.

15. Wurtz P, Soininen P, Kangas AJ, et al. Branched-chain and aromatic amino acids are predictors of insulin resistance in young adults. Diabetes Care 2013;36:648-55.

16. Palmer ND, Stevens RD, Antinozzi PA, et al. Metabolomic profile associated with insulin resistance and conversion to diabetes in the Insulin Resistance Atherosclerosis Study. J Clin Endocrinol Metab 2015;100:E463-8.

17. Stancakova A, Civelek M, Saleem NK, et al. Hyperglycemia and a common variant of GCKR are associated with the levels of eight amino acids in 9,369 Finnish men. Diabetes 2012;61:1895-902.

18. Wang TJ, Larson MG, Vasan RS, et al. Metabolite profiles and the risk of developing diabetes. Nat Med 2011;17:448-53.

19. Butte NF, Liu Y, Zakeri IF, et al. Global metabolomic profiling targeting childhood obesity in the Hispanic population. Am J Clin Nutr 2015;102: 256-67.

20. Perng W, Gillman MW, Fleisch AF, et al. Metabolomic profiles and childhood obesity. Obesity (Silver Spring) 2014;22:2570-8.

21. McCormack SE, Shaham O, McCarthy MA, et al. Circulating branchedchain amino acid concentrations are associated with obesity and future insulin resistance in children and adolescents. Pediatr Obes 2013;8:52-61.

22. Michaliszyn SF, Sjaarda LA, Mihalik SJ, et al. Metabolomic profiling of amino acids and beta-cell function relative to insulin sensitivity in youth. J Clin Endocrinol Metab 2012;97:E2119-24.

23. Mihalik SJ, Michaliszyn SF, de las Heras J, et al. Metabolomic profiling of fatty acid and amino acid metabolism in youth with obesity and type 2 diabetes: evidence for enhanced mitochondrial oxidation. Diabetes Care 2012;35:605-11.

24. Pardridge WM, Choi TB. Neutral amino acid transport at the human blood-brain barrier. Fed Proc 1986;45:2073-8.

25. Fernstrom JD. Branched-chain amino acids and brain function. J Nutr 2005; 135:1539s-46s.

26. Fernstrom JD. Aromatic amino acids and monoamine synthesis in the central nervous system: influence of the diet. J Nutr Biochem 1990;1:508-17.
27. Coppola A, Wenner BR, Ilkayeva O, et al. Branched-chain amino acids alter neurobehavioral function in rats. Am J Physiol Endocrinol Metab 2013;304:E405-13.

28. Canadian Institute for Health Information., \& Public Health Agency of Canada Obesity in Canada: a joint report from the Public Health Agency of Canada and the Canadian Institute for Health Information, 2011. Available at http://www.phac-aspc.gc.ca/hp-ps/hl-mvs/oic-oac/index-eng. php (accessed 12 October 2017).

29. Boucher O, Jacobson SW, Plusquellec P, et al. Prenatal methylmercury, postnatal lead exposure, and evidence of attention deficit/hyperactivity disorder among Inuit children in Arctic Quebec. Environ Health Perspect 2012;120:1456-61.

30. Dallaire F, Dewailly É, Muckle G, Ayotte P. Time trends of persistent organic pollutants and heavy metals in umbilical cord blood of inuit infants born in Nunavik (Québec, Canada) between 1994 and 2001. Environ Health Perspect 2003;111:1660-4.

31. Muckle G, Ayotte P, Dewailly EE, Jacobson SW, Jacobson JL. Prenatal exposure of the northern Quebec Inuit infants to environmental contaminants. Environ Health Perspect 2001;109:1291-9.

32. Achenbach TM, Rescorla LA. Manual for the ASEBA School-Age Forms \& Profiles: An Integrated System of Multi-Informant Assessement. Burlington, VT: University of Vermont, Research Center for Children, Youth \& Families, 2001:1-9.

33. Roy C, Tremblay PY, Bienvenu JF, Ayotte P. Quantitative analysis of amino acids and acylcarnitines combined with untargeted metabolomics using ultra-high performance liquid chromatography and quadrupole time-of-flight mass spectometry. J Chromatogr B Analyt Technol Biomed Life Sci 2016;1027:40-9.

34. Medehouenou TC, Ayotte P, St-Jean A, et al. Overweight and obesity prevalence among school-aged Nunavik Inuit children according to three body mass index classification systems. J Adolesc Health 2015;57:31-6.

35. Cole TJ, Bellizzi MC, Flegal KM, Dietz WH. Establishing a standard definition for child overweight and obesity worldwide: international survey. BMJ 2000;320:1240-3.

36. Centers for Disease Control and Prevention A SAS program for the CDC grow charts (ages 0 to $<20$ years), 2015. Available at http://www.cdc. gov/nccdphp/dnpao/growthcharts/resources/sas.htm (accessed 24 August 2017).

37. Hollingshead AB. Four factor index of social status. New Haven, CT: Yale University Department of Sociology, 1975.

38. van Spronsen FJ, Hoeksma M, Reijngoud DJ. Brain dysfunction in phenylketonuria: is phenylalanine toxicity the only possible cause? J Inherit Metab Dis 2009;32:46-51.

39. Albert PR, Benkelfat C, Descarries L. The neurobiology of depressionrevisiting the serotonin hypothesis. I. Cellular and molecular mechanisms. Philos Trans R Soc Lond B Biol Sci 2012;367:2378-81.

40. Hamon M, Blier P. Monoamine neurocircuitry in depression and strategies for new treatments. Prog Neuropsychopharmacol Biol Psychiatry 2013;45:54-63. 